\title{
STABILISATION DE L'ÉQUATION DE LA CHALEUR COMMANDÉE EN FLUX
}

\author{
JEAN-JACQUES LOISEAU ET HUGUES MOUNIER
}

\begin{abstract}
RÉSUMÉ. On considère une barre finie, chauffée à une extrêmité, la température de l'autre extrêmité étant mesurée. Des méthodes sont proposées pour stabiliser le système, autrement dit déterminer le flux de chaleur en fonction de la température de façon à ce que la température atteigne asymptotiquement une valeur prescrite. Une approche symbolique est utilisée. Il est bien connu que l'équation de la chaleur à une dimension d'espace conduit à un transfert qui est rationnel en la racine carrée de la variable de Laplace et en son exponentielle. Cela permet d'adapter des méthodes connues de stabilisation des systèmes à retards. Les lois de commande obtenues sont convolutives: le flux de chaleur est déterminé par une convolution de la température et, éventuellement, de sa dérivée.
\end{abstract}

ABstract. A heat conducting rod of finite length is considered. This rod is heated at one end, and the temperature of the other end is measured. Methods are proposed to calculate a control law, i.e. express the heat flux as a function of the temperature, so that the closed-loop system is stable, i.e. the temperature asymptotically reaches a given one. The operational approach is used. The 1-D heat equation indeed gives rise to a transfer that is rational in the square root of the Laplace variable and its exponential. This permits to adapt the known constructive methods for the stabilisation of time-delay systems, that are recalled. The resulting control laws are convolutive. The heat flux is determined via a convolution from the measured temperature or its derivative.

\section{INTRODUCTION}

L'objectif de cette communication est de montrer que des techniques récemment développées pour la stabilisation des systèmes à retards permettent la synthèse de bouclage stabilisants pour une classe de systèmes fractionnaires. L'exemple traité est celui de l'équation de la chaleur commandée en flux. On considère une barre homogène, isolée, conduisant la chaleur, chauffée en une extrêmité et on cherche à stabiliser la température de l'autre extrêmité en asservissant le flux de chaleur à la mesure de température.

Les outils que nous privilégions pour ce type de question sont de nature algébrique. Pour une large classe de systèmes, la synthèse d'un bouclage stabilisant se ramène à la solution d'une équation Diophantienne —ou d'une identité de Bézoutsur un anneau. Dans le cas des systèmes linéaires invariants dans le temps de dimension finie, l'anneau de référence est simplement l'anneau des polynômes en une

(C) Société de Mathématiques Appliquées et Industrielles. Typeset by LATEX.

J.J. Loiseau: Institut de Recherche en Cybernétique de Nantes, UMR CNRS 6597, 1 rue de la Noë, BP 92101, 44321 Nantes Cedex 03, France. E-mail: loiseau@ircyn.prd.fr.

H. Mounier: Institut d'Electronique Fondamentale, Université Paris sud, 91405 Orsay, France. Email: mounier@ief.u-psud.fr.

Les auteurs remercient D. Brethé, M. Cardelli, P. Cosson, R. Hotzel and V. Kharitonov, pour les commentaires qu'ils leur ont prodigués durant la préparation de ce travail, D. Matignon et G. Montseny pour la révision du manuscrit. 
variable à coefficients réels, qui est un domaine de Bézout, et les solutions sont bien connues. Elles ont été écrites sous des formes très variées, approches polynômiales, géométrique, modules, factorisations propres et stables et ont donné lieu à de très nombreux développements, stabilisation par divers types de lois de commande, rejet de perturbation, robustesse, (voir par exemple, choisis parmi des ouvrages de référence en automatique [21, 31, 34, 35]).

Un formalisme plus général, qui s'appuit sur la définition d'anneaux de convolutions, a été introduit par divers auteurs [7, 8, 17, 18], mais les techniques proposées ne sont pas toujours constructives. Pour ce qui concerne les systèmes à retards commensurables, l'introduction de l'anneau $\mathcal{E}[3,13]$ des fractions rationnelles en $s, \mathrm{e}^{-s}$ qui sont analytiques en $s$ a récemment permis d'obtenir une construction explicite de bouclage stabilisant, qui généralise et formalise une construction classique pour les systèmes à retards sur l'entrée $[23,28]$ Tout repose sur le fait qu' $\mathcal{E}$ est un domaine de Bézout [4], et qu'en conséquence, une procédure constructive de solution d'équation Diophantiennees est décrite. La loi de commande ainsi calculée est un système à retards, qui inclut des retards localisés, mais aussi en général des retards distribués (convolutions à noyau régulier à support compact). Cette procédure s'étend au cas des systèmes à retards de type neutre formellement stable [2]. Dans le cas de systèmes non formellement stable, un bouclage sur la dérivée de la mesure permet encore de stabiliser [6, 27]. Ces résultats récents concernant la stabilisation forment, avec ceux concernant la poursuite de trajectoire en boucle ouverte $[10,11,12,26]$, une théorie assez complète de la commande des systèmes à retards commensurables.

Ainsi qu'il est bien connu (voir par exemple [9, 15, 33], l'équation de la chaleur admet un transfert qui est rationnel en la racine carrée de la variable de Laplace, $\sqrt{s}$ et en son exponentielle, $\mathrm{e}^{-\sqrt{s}}$. C'est ce qui autorise l'utilisation des résultats concernant les systèmes à retards.

Dans le second paragraphe, nous présentons la solution symbolique, exprimée en termes de transformée de Laplace, de l'équation de la chaleur. Dans le troisième paragraphe, nous présentons les outils de base utilisés pour calculer des bouclages stabilisants et nous illustrerons ces techniques dans le cas de l'équation de la chaleur dans le paragraphe quatre.

\section{L'ÉQUATION DE LA CHALEUR}

\subsection{Position du PRoBlÈme}

On considère une barre uniforme, homogène, isolée, conduisant la chaleur et de longueur finie $l$. Cette barre est chauffée à l'extrêmité d'abscisse $x=l$. La température dans cette barre est une fonction $T(x, t)$ de l'abscisse $x, 0 \leq x \leq l$, et du temps $t, t \geq 0$, solution de l'équation de la chaleur

$$
h^{2} \frac{\partial T(x, t)}{\partial t}=\frac{\partial^{2} T(x, t)}{\partial x^{2}}
$$

avec les conditions aux limites de type Newman

$$
\begin{aligned}
& \frac{\partial T(0, t)}{\partial x}=0 \\
& \frac{\partial T(l, t)}{\partial x}=u(t)
\end{aligned}
$$

où $u(t)$ désigne le flux de chaleur qui est la variable de commande du système, $h^{2}$ est un coefficient de conduction positif. La température mesurée à la seconde extrêmité, d'abscisse $x=0$, est la sortie du système, qu'on note $y(t)$

$$
y(t)=T(0, t)
$$

ESAIM: Proc., VOL. 5, 1998, 131-144 
Dans ces conditions, la température $T(x, t)$ dépend linéairement de l'entrée $u(t), 0 \leq$ $t$, et des conditions initiales de température dans la barre, $T(x, 0), 0 \leq x \leq l[9]$. Lorsque la température initiale est constante, $T(x, 0)=T_{0}, 0 \leq x \leq l$, où $\bar{T}_{0} \in \mathbb{R}$, la différence $T(x, 0)-T_{0}$ dépend linéairement de l'entrée $u$. On supposera dans la suite que

$$
T_{0}=0,
$$

ce qui n'est pas limitatif pour le présent propos.

\subsection{Formulation symbolique}

La solution du système entrée-sortie (2.1-5) s'exprime élégamment en termes de transformées de Laplace. Une fonction $f(x, t), t \geq 0$ étant donnée, sa transformée de Laplace temporelle [15], notée $\mathcal{L} f(x, t)$ ou plus simplement $\hat{f}(x, s)$, est définie par

On a alors

$$
\mathcal{L}(f(x, t))=\hat{f}(x, s)=\int_{0}^{\infty} f(x, t) \mathrm{e}^{-s t} d t
$$

$$
\mathcal{L}\left(\frac{\partial T(x, t)}{\partial t}\right)=s \mathcal{L}(T(x, t))-T(x, 0)
$$

ainsi que

$$
\mathcal{L}\left(\frac{\partial^{2} T(x, t)}{\partial x^{2}}\right)=\frac{\partial}{\partial x^{2}} \mathcal{L}(T(x, t)),
$$

et le système (2.1) devient

$$
\frac{\partial^{2}}{\partial x^{2}} \hat{T}(x, s)=h^{2} s \hat{T}(x, s)-h^{2} T(x, 0),
$$

qui est en fait une équation différentielle ordinaire en la variable $x$, dont l'équation caractéristique

$$
\lambda^{2}-h^{2} s=0
$$

a deux solutions, $\lambda= \pm h \sqrt{s}$, de sorte que la solution générale, autrement dit, sous l'hypothese (2.5), la solution cherchée, s'écrit

$$
\hat{T}(x, s)=T_{1}(s) \mathrm{e}^{h x \sqrt{s}}+T_{2}(s) \mathrm{e}^{-h x \sqrt{s}}
$$

On en déduit en particulier que

$$
\frac{\partial}{\partial x} \hat{T}(x, s)=h \sqrt{s}\left(T_{1}(s) \mathrm{e}^{h x \sqrt{s}}-T_{2}(s) \mathrm{e}^{-h x \sqrt{s}}\right) .
$$

La condition aux limites (2.2) se reécrit sous forme symbolique

$$
\frac{\partial}{\partial x} \hat{T}(0, s)=0,
$$

d'où l'on déduit que $T_{1}(s)=T_{2}(s)$, quant à la condition (2.3), elle devient

et on en déduit

$$
\frac{\partial}{\partial x} \hat{T}(l, s)=\hat{u}(s)
$$

pour $i=1,2$, de sorte que finalement

$$
h \sqrt{s} T_{i}(s)\left(\mathrm{e}^{h l \sqrt{s}}-\mathrm{e}^{-h l \sqrt{s}}\right)=\hat{u}(s),
$$

$$
\hat{T}(x, s)=\frac{\mathrm{e}^{h x \sqrt{s}}+\mathrm{e}^{-h x \sqrt{s}}}{h \sqrt{s}\left(\mathrm{e}^{h l \sqrt{s}}-\mathrm{e}^{-h l \sqrt{s}}\right)} \hat{u}(s)
$$

et en particulier

$$
\hat{y}(s)=\frac{2 \mathrm{e}^{-h l \sqrt{s}}}{h \sqrt{s}\left(1-\mathrm{e}^{-2 h l \sqrt{s}}\right)} \hat{u}(s)
$$


On appelle transfert de ce système la fonction

$$
\hat{H}(s)=\frac{2 \mathrm{e}^{-h l \sqrt{s}}}{h \sqrt{s}\left(1-\mathrm{e}^{-2 h l \sqrt{s}}\right)}
$$

Il s'agit maintenant de calculer le flux injecté $u(t), t \geq 0$, en fonction de la température $y(t), t \geq 0$, afin que cette dernière soit asymptotiquement stable autour d'une valeur prescrite. Bien entendu il est requis que la stabilité soit effective, la convergence de $y(t)$ n'est pas affectée par un bruit sur la mesure de température et que la loi de commande puisse être implantée en ligne, $u(t)$ ne dépendant que des valeurs $y(\tau)$ pour $0 \leq \tau \leq t$. Il convient d'abord d'introduire succintement quelques-uns des concepts qui permettent la solution de ce type de questions.

\section{Quelques Éléments de la théorie de la stabilisation des SYSTÈMES LINÉAIRES}

\subsection{Convolutions}

Un système causal défini par une convolution est une relation entrée-sortie de la forme $y=T * u$, autrement dit

$$
y(t)=\int_{0}^{t} T(t-\tau) u(\tau) d \tau,
$$

où $T(t)$, le noyau de la convolution, $u(t)$, l'entrée du système et $y(t)$, la sortie du système, sont des fonctions généralisées ou distributions dont le support est $\mathbb{R}_{+}$. L. Schwartz [32] a montré que tout opérateur linéaire continu (au sens des distributions), causal et invariant par translation, admet une représentation de ce type. Par transformation de Laplace, au sens des distributions tempérées, le système devient

$$
\hat{y}(s)=\hat{T}(s) \hat{u}(s),
$$

on dit alors que $\hat{T}(s)$ est le transfert de ce système.

L'équation de la chaleur est un exemple de système de ce type. On peut, en vue d'exprimer $y(t)$ en fonction de $u(t)$, calculer le noyau du transfert $\hat{H}(s)$ qui apparait dans l'équation (2.6), mais ce n'est pas très simple, et ce n'est pas la meilleure façon de calculer numériquement $y(t)$. C'est de toute façon inutile en vue de l'objectif qui est de stabiliser l'équation de la chaleur. Par ailleurs, dans le contexte de la présente étude, le cadre des fonctions généralisées est trop large. Nous allons maintenant introduire des concepts qui conviennent mieux à notre propos.

\subsection{L'algèbre de convolutions $\mathcal{R}(\sigma)[7,8]$}

Définition 3.1. Etant donné $\sigma \in \mathbb{R}$, on définit $\mathcal{R}(\sigma)$ comme l'ensemble des fonctions généralisées de la forme

$$
f(t)= \begin{cases}0 & , t<0 \\ f_{a}(t)+f_{a p}(t) & , t \geq 0\end{cases}
$$

où $\mathrm{e}^{-\sigma t} f_{a}(t)$ est mesurable, telle que

$$
\int_{0}^{\infty}\left|\mathrm{e}^{-\sigma t} f_{a}(t)\right| d t<\infty
$$

et où

$$
f_{a p}=\sum_{i=0}^{\infty} f_{i} \delta_{t_{i}},
$$

ESAIM: Proc., VOL. 5, 1998, 131-144 
avec $f_{i} \in \mathbb{C}, 0=t_{0}, t_{i} \geq 0, i \geq 1, \delta_{t_{i}}$ désigne la distribution de Dirac en $t_{i}$, et

$$
\sum_{i=0}^{\infty} \mathrm{e}^{-\sigma t_{i}}\left|f_{i}\right|<\infty
$$

$\mathcal{R}(\sigma)$, muni de l'addition et du produit de convolution, est un anneau commutatif intègre [18] qui contient l'élément neutre $\delta_{0}$, et c'est une $\mathbb{C}$-algèbre de Banach commutative [8] avec la norme définie par

$$
\|f(t)\|_{\mathcal{R}(\sigma)}=\left\|\mathrm{e}^{-\sigma t} f_{a}(t)\right\|_{1}+\sum_{i \in \mathbb{N}} \mathrm{e}^{-\sigma t_{i}}\left|f_{i}\right| .
$$

L'ensemble $\hat{\mathcal{R}}(\sigma)$ des transformées de Laplace d'éléments de $\mathcal{R}(\sigma)$, muni du produit ordinaire des fonctions, est également un anneau commutatif intègre, dont l'élément neutre est 1 et une $\mathbb{C}$-algèbre de Banach commutative pour la topologie image.

ThÉorème 3.2. $[7,8]$ Soit $\hat{f}$ un élément de $\hat{\mathcal{R}}(\sigma)$. Alors

(i) $\hat{f}$ est analytique dans la partie du plan complexe à droite de $\sigma$.

(ii) $\hat{f}$ est bornée dans la partie du plan complexe à droite de $\sigma$

$$
\sup _{\operatorname{Re}(s) \geq \sigma}|\hat{f}(s)|<\|f\|_{\mathcal{R}(\sigma)} .
$$

(iii) $\hat{f}$ possède une inverse dans $\hat{\mathcal{R}}(\sigma)$ si et seulement si

$$
\inf _{\operatorname{Re}(s) \geq \sigma}|\hat{f}(s)|>0 .
$$

Ce résultat est important en pratique. Il caractérise les éléments inversibles de $\hat{\mathcal{R}}(\sigma)$, donc aussi les éléments inversibles de $\mathcal{R}(\sigma)$. Le problème est que cette formulation en termes de modules n'est guère utilisable. Nous lui préférons la formulation suivante $[7,8]$.

THÉorème 3.3. $\hat{f}$ possède une inverse dans $\hat{\mathcal{R}}(\sigma)$ si et seulement si $\hat{f}$ ne possède pas de zéro dans la partie du plan complexe à droite de $\sigma$, même à l'infini. Autrement dit les deux conditions suivantes sont vérifiées

(i) $\quad \hat{f}(s)=0 \Longrightarrow \operatorname{Re}(s)<\sigma$,

(ii) $\quad$ Si $\hat{f}\left(s_{k}\right)$ est une suite de Cauchy, $\operatorname{Re}\left(s_{k}\right) \geq \sigma,\left|s_{k}\right| \rightarrow \infty$, alors $\lim \hat{f}\left(s_{k}\right)$ est non nulle.

Un exemple typique ne satisfaisant pas la condition (ii) est

$$
\hat{f}(s)=1-\mathrm{e}^{-s}+\frac{1}{s+1},
$$

car $\lim _{k \rightarrow \infty} \hat{f}(2 j k \pi)=0$.

Dans le cas de l'équation de la chaleur par exemple, on peut constater que $1-\mathrm{e}^{-2 h l \sqrt{s}}$ est un élément inversible de $\hat{\mathcal{R}}(\sigma)$ pour tout $\sigma$ strictement positif. C'est pourquoi l'équation (2.6) définit bien $y(t)$ en fonction de $u(t)$. Plutôt qu'une expression explicite, il vaut mieux exprimer $y(t)$ de façon implicite au moyen d'une équation de Volterra. Du fait que [15]

$$
\mathcal{L}^{-1}\left(\mathrm{e}^{-2 h l \sqrt{s}}\right)=\frac{h l}{t \sqrt{\pi t}} \mathrm{e}^{-\frac{h^{2} l^{2}}{t}}
$$

et que

$$
\mathcal{L}^{-1}\left(\frac{2 \mathrm{e}^{-h l \sqrt{s}}}{h \sqrt{s}}\right)=\frac{2}{h \sqrt{\pi t}} \mathrm{e}^{-\frac{h^{2} l^{2}}{4 t}},
$$

on obtient

$$
y(t)=\int_{0}^{t} \frac{h l}{\tau \sqrt{\pi \tau}} \mathrm{e}^{-\frac{h^{2} l^{2}}{\tau}} y(t-\tau) d \tau+\int_{0}^{t} \frac{2}{h \sqrt{\pi \tau}} \mathrm{e}^{-\frac{h^{2} l^{2}}{4 \tau}} u(t-\tau) d \tau .
$$


Cette expression diverge lorsque $t$ grandit (si par exemple $u(t)$ est une constante positive). Cela traduit le fait que le système n'est pas asymptotiquement stable, ce qu'on peut relier au fait que

et

$$
\frac{1}{1-\mathrm{e}^{-2 h l \sqrt{s}}}
$$

$$
\frac{2 \mathrm{e}^{-h l \sqrt{s}}}{h \sqrt{s}}
$$

ne sont des éléments de $\hat{\mathcal{R}}(\sigma)$ que pour $\sigma$ strictement positif.

\subsection{Stabilité}

On dit que le système (3.1) est $L_{p}$-stable, $p$ étant un entier positif, si pour toute entrée $u \in L_{p}$, autrement dit $u$ est localement intégrable et

$$
\int_{0}^{\infty}|u(t)|^{p} d t<\infty
$$

alors la sortie $y$ du système est aussi dans $L_{p}$. Rappellons que pour une telle fonction $u$, on définit

$$
\|u\|_{p}=\left(\int_{0}^{\infty}|u(t)|^{p} d t\right)^{\frac{1}{p}}
$$

THÉo RÈme 3.4. [8] Le système (3.1) est $L_{p}-$ stable, pour $1 \leq p \leq \infty$ si et seulement si $\hat{T}(s) \in \hat{\mathcal{R}}(0)$.

Cela provient de l'inégalité

$$
\|T * u\|_{p} \leq\|T\|_{\mathcal{R}(0)}\|u\|_{p}
$$

qui peut être une égalité pour certaines fonctions $u$ et pour $p=1$ ou $p=\infty$.

Dans le cas de l'équation de la chaleur et, comme nous le verrons par la suite, dans le cas de systèmes bouclés, il est important de formuler un critère de stabilité qui prenne en compte que le transfert est défini par une fraction.

Corollaire 3.5. Supposons que $\hat{T}(s)=\hat{N}(s) \hat{D}^{-1}(s)$, où $\hat{N}(s), \hat{D}(s) \in \hat{\mathcal{R}}(0)$ Alors le système de transfert $\hat{T}(s)$ est $L_{p}$-stable, pour $1 \leq p \leq \infty$ si et seulement si $\hat{D}(s)$ n'a pas de zéro dans la partie droite du plan complexe, même à l'infini.

Ce résultat est bien connu dans de nombreux cas particuliers, systèmes à retards [1, 20], systèmes fractionnaires [24], systèmes fractionnaires à retards [16]. L'équation de la chaleur n'appartient à aucune de ces catégories. Elle est instable du fait que le dénominateur de son transfert s'annule pour $s=0$.

\subsection{Système Bouclé PaR UNE LOI DE COMMANDE}

On parle de loi de commande lorsqu'on détermine $u(t)$ en fonction de $y(t)$ et d'une nouvelle entrée $v(t)$, qui peut être une consigne - par exemple de température, dans le cas de l'équation de la chaleur - ou une trajectoire qu'on souhaite voir suivre au plus près par la sortie $y(t)$, par exemple. Pour notre propos nous choisirons une loi de commande de la forme

$$
\hat{u}(s)=\hat{F}(s)(\hat{v}(s)-\hat{y}(s))
$$

où $\hat{F}(s)$ est dans $\hat{\mathcal{R}}(\sigma)$ pour au moins un réel $\sigma$. Le système bouclé peut être perturbé par un signal $w(t)$ se superposant à l'entrée $u(t)$, par exemple l'erreur numérique effectuée lors de l'évaluation de $u(t)$ par la loi (3.2), des aléas de fonctionnement de l'actionneur par lequel on réalise $u(t)$, du système de chauffage dans le cas de l'équation de la chaleur. Le système (3.1) devient alors

$$
\hat{y}(s)=\hat{T}(s)(\hat{u}(s)+\hat{w}(s)) .
$$


Le système bouclé (3.2-3.3) s'écrit sous forme matricielle

$$
\begin{aligned}
\left(\begin{array}{l}
\hat{y}(s) \\
\hat{u}(s)
\end{array}\right)= & \left(\begin{array}{cc}
0 & \hat{T}(s) \\
-\hat{F}(s) & 0
\end{array}\right)\left(\begin{array}{l}
\hat{y}(s) \\
\hat{u}(s)
\end{array}\right)+\left(\begin{array}{cc}
0 & \hat{T}(s) \\
\hat{F}(s) & 0
\end{array}\right)\left(\begin{array}{c}
\hat{v}(s) \\
\hat{w}(s)
\end{array}\right) \\
= & \left(\begin{array}{cc}
1 & -\hat{T}(s) \\
\hat{F}(s) & 1
\end{array}\right)^{-1}\left(\begin{array}{cc}
0 & \hat{T}(s) \\
\hat{F}(s) & 0
\end{array}\right)\left(\begin{array}{c}
\hat{v}(s) \\
\hat{w}(s)
\end{array}\right) \\
= & \left(\begin{array}{c}
\hat{T}(s) \\
1
\end{array}\right)(1+\hat{F}(s) \hat{T}(s))^{-1}\left(\begin{array}{ll}
\hat{F}(s) & 1
\end{array}\right)+ \\
& \left.\left(\begin{array}{cc}
0 & 0 \\
0 & -1
\end{array}\right)\right)\left(\begin{array}{l}
\hat{v}(s) \\
\hat{w}(s)
\end{array}\right)
\end{aligned}
$$

\subsection{STABILISATION}

On dit que le système bouclé (3.2-3.3) est stable lorsque les quatres transferts, entre les deux entrées $v$ et $w$ et les deux sorties $u$ et $y$ sont stables. Lorsque le système en boucle ouverte et la loi de commande sont tous deux décrits sous forme de fractions déléments de $\hat{\mathcal{R}}(0)$,

$$
\hat{T}(s)=\hat{N}(s) \hat{D}^{-1}(s), \hat{F}(s)=\hat{Q}^{-1}(s) \hat{P}(s),
$$

$\hat{D}(s)$ et $\hat{Q}(s)$ étant inversibles dans $\hat{\mathcal{R}}(\sigma)$, ce transfert matriciel s'écrit

$$
\left(\begin{array}{c}
\hat{N}(s) \\
\hat{D}(s)
\end{array}\right)(\hat{Q}(s) \hat{D}(s)+\hat{P}(s) \hat{N}(s))^{-1}\left(\begin{array}{ll}
\hat{P}(s) & \hat{Q}(s)
\end{array}\right)+\left(\begin{array}{cc}
0 & 0 \\
0 & -1
\end{array}\right)
$$

ainsi

$$
\hat{X}(s)=\hat{Q}(s) \hat{D}(s)+\hat{P}(s) \hat{N}(s)
$$

est le dénominateur du système bouclé.

Corollaire 3.6. Dans le cas où $\hat{N}(s), \hat{D}(s), \hat{P}(s)$, et $\hat{Q}(s)$ sont dans $\hat{\mathcal{R}}(0)$, le système bouclé (3.2-3.3) est $L_{p}-$ stable, pour $1 \leq p \leq \infty$ si et seulement si

$$
\inf _{\operatorname{Re}(s) \geq 0}|\hat{Q}(s) \hat{D}(s)+\hat{P}(s) \hat{N}(s)|>0,
$$

autrement dit si $\hat{Q}(s) \hat{D}(s)+\hat{P}(s) \hat{N}(s)$ n'a pas de zéro dans la partie droite du plan complexe, même à l'infini.

Le problème de stabilisation est maintenant assez bien posé. Il s'agit de calculer les transferts $\hat{P}(s), \hat{Q}(s) \in \hat{\mathcal{R}}(0)$, de façon à ce que la condition du corollaire 3.6 soit satisfaite. Il faut aussi que $\hat{Q}(s)$ soit un élément inversible de $\hat{\mathcal{R}}(\sigma)$, pour quelque $\sigma \in \mathbb{R}$, autrement dit

$$
\inf _{\operatorname{Re}(s) \geq \sigma}|\hat{Q}(s)|>0,
$$

pour que $\hat{F}(s) \in \hat{\mathcal{R}}(\sigma)$. Du fait de la définition 3.1, de $\mathcal{R}(\sigma), \hat{Q}(s)$ s'écrit

$$
\hat{Q}(s)=f_{0}+\sum_{i=1}^{\infty} f_{i} \mathrm{e}^{-s t_{i}}+\hat{f}_{a}(s),
$$

où $t_{i}>0, i>1$, et $f_{a}(t)$ est localement intégrable. Finalement il existe un tel $\sigma$ si et seulement si $f_{0} \neq 0$, autrement dit si

$$
\lim _{\operatorname{Re}(s) \rightarrow \infty} \hat{Q}(s) \neq 0 .
$$

On peut reformuler cette condition en termes de primarité, ainsi qu'il est classique de le faire dans le cas de systèmes de dimension finie. 
Corollaire 3.7. Si $\hat{N}(s), \hat{D}(s) \in \hat{\mathcal{R}}(0)$, le système est stabilisable si $\exists \hat{P}(s), \hat{Q}(s) \in$ $\hat{\mathcal{R}}(0)$ tels que

$$
\begin{aligned}
& \text { (i) } \hat{Q}(s) \hat{D}(s)+\hat{P}(s) \hat{N}(s)=1, \\
& \text { (ii) } \lim _{|s| \geq 0, \operatorname{Re}(s) \rightarrow \infty} \hat{Q}(s) \neq 0 .
\end{aligned}
$$

En effet, $\hat{Q}(s) \hat{D}(s)+\hat{P}(s) \hat{N}(s)=\hat{X}(s)$ est un élément inversible de $\hat{\mathcal{R}}(0)$, donc $\hat{X}^{-1}(s) \hat{Q}(s), \hat{X}^{-1}(s) \hat{P}(s) \in \hat{\mathcal{R}}(0)$. Ils satisfont l'identité de Bézout du corollaire. De plus $\hat{Q}^{-1}(s) \hat{P}(s) \in \hat{\mathcal{R}}(\sigma)$, pour quelque $\sigma \geq 0$.

La condition du corollaire est en fait nécessaire et suffisante pour assurer la stabilité $L_{p}, 1 \leq p \leq \infty$ (ce résultat apparait sous une forme voisine dans [8], $\mathrm{p}$. 86). Elle n'est pas toujours nécessairement satisfaite si $p$ est fixé.

\subsection{LE CAS DES SYSTÈMES À RETARDS}

Les systèmes à retards sont des systèmes dont le transfert est rationnel en les variables $s$ et $\mathrm{e}^{\theta_{i} s}$, où $\theta_{i} \in \mathbb{R}, i=1,2, \ldots$ Les conditions pour leur stabilité sont bien connues.

THÉoRÈME 3.8. $[1,20]$ Un système à retards dont le transfert est $\hat{T}(s)=\hat{B}(s) \hat{A}^{-1}(s)$, $\hat{B}(s)$ et $\hat{A}(s)$ étant des polynômes en $s$, $\mathrm{e}^{\theta_{i} s}$, est stable si et seulement si il existe $\alpha \in \mathbb{R}, \alpha<0$, tel que $\hat{A}(s)=0 \Rightarrow \operatorname{Re}(s)<\alpha$.

Si $\theta$ est le plus grand des $\theta_{i}$, et $n$ est le degré en la variable $s$ du dénominateur $\hat{A}(s)$, qui est plus grand que le degré en la variable $s$ du numérateur $\hat{B}(s)$ si le transfert est un élément de $\hat{\mathcal{R}}(\sigma)[7]$, on déduit facilement une nouvelle factorisation du transfert en termes d'éléments de $\hat{\mathcal{R}}(0)$ comme dans le corollaire 3.5

$$
\begin{aligned}
& \hat{N}(s)=\frac{\mathrm{e}^{-\theta s} \hat{B}(s)}{(s+1)^{n}}, \\
& \hat{D}(s)=\frac{\mathrm{e}^{-\theta s} \hat{A}(s)}{(s+1)^{n}} .
\end{aligned}
$$

Il est bien clair que les zéros instables de $\hat{A}(s)$ et de $\hat{D}(s)$ sont identiques. Les conditions du théorème 3.8 sont en fait équivalentes, dans le cas d'un système à retards, aux conditions du corollaire 3.5. De la même façon, le corollaire 3.6 peut s'exprimer en ces termes.

ThÉorème 3.9. Avec les notations précédentes, le sytème à retards de transfert $\hat{T}(s)$ est stabilisable si et seulement si le numérateur $\hat{B}(s)$ et le dénominateur $\hat{A}(s)$, ou, de façon équivalente, $\hat{N}(s)$ et $\hat{D}(s)$ n'ont pas de zéros communs à partie réelle positive ou nulle, même à l'infini, autrement dit, pour toute suite $s_{k}, \operatorname{Re}\left(s_{k}\right) \geq 0$, $\left|s_{k}\right| \rightarrow \infty$ telle que $\hat{N}\left(s_{k}\right)$ et $\hat{D}\left(s_{k}\right)$ sont des suites de Cauchy, alors $\lim \hat{N}\left(s_{k}\right)$ ou $\lim \hat{D}\left(s_{k}\right)$ est non nulle.

D'un point de vue pratique, il n'existe pas de méthodologie générale pour la synthèse des lois de commande stabilisantes pour les systèmes à retards quelconques. En revanche une telle méthode de conception existe dans le cas particuliers où les retards du système sont commensurables.

\subsection{Le Cas des Systèmes À Retards COMmensurables: L'anneau $\mathcal{E}$}

Le transfert d'un système à retards dont les retards sont tous commensurables à $\theta, \theta>0$, est rationnel en les deux variables $s$ et $\mathrm{e}^{-\theta s}$. C'est par exemple le cas du transfert de l'équation

$$
\dot{x}(t)=u(t)-u(t-\theta)
$$

ESAIM: Proc., VOL. 5, 1998, 131-144 
dont le transfert est

$$
\hat{T}(s)=\frac{1-\mathrm{e}^{-\theta s}}{s} .
$$

Ce transfert est la transformée de Laplace d'un noyau causal à support compact,

$$
T(t)=\left\{\begin{array}{cc}
0 & t<0, \\
1 & 0<t<\theta \\
0 & t>\theta .
\end{array}\right.
$$

Un tel transfert est appelé transformée de Laplace finie. Le système correspondant est appelé un retard distribué. L'emploi de ce type de convolutions s'est révélé efficace pour stabiliser les systèmes à retards. Elles ont d'abord été utilisées dans le cas de systèmes avec des retards sur l'entrée [28, 29], puis pour une classe de systèmes comportant aussi des retards sur l'état [23]. La méthode a été généralisée dans [19] et des résultats récents $[4,13]$ concernant la structure algébrique de cette classe de convolutions ont finalement conduit à une procédure constructive et générale pour la stabilisation des systèmes à retards [5, 3].

DÉFinition 3.10. On appelle $\mathcal{G}$ l'ensemble des transformées de Laplace finies qui sont rationnelles en $s, \mathrm{e}^{-\theta s}$. On appelle $\mathcal{E}$ l'ensemble $\mathcal{G}[s]$ des polynômes en $s$ à coefficients dans $\mathcal{G}$.

$\mathcal{E}$ est un anneau intègre, autrement dit il contient l'élément neutre 1 , est commutatif et n'a pas de diviseur de zéro. Les notions de diviseur ou de multiple sont définis classiquement, mais pourtant $\mathcal{E}$ n'est pas un domaine factoriel, un élément $x(s)$ de $\mathcal{E}$ possédant en général un nombre infini de facteurs irréductibles, qui sont des polynômes la forme $s-\alpha, \alpha$ étant un zéro réel de $x(s)$, ou $s^{2}-\beta^{2}+\gamma$ pour les zéros complexes conjugués. $\mathcal{E}$ n'est pas non plus un anneau Noethérien, pour la même raison. Il possède cependant des propriétés qui le rendent manipulable.

Définition 3.11. Deux éléments $x(s), y(s) \in \mathcal{E}$ sont dit premiers s'ils n'ont pas de facteur commun, hormis des constantes non nulles, autrement dit s'ils n'ont pas de zéro commun.

ThÉorème 3.12. [4] L'anneau $\mathcal{E}$ est un domaine de Bézout. Autrement dit, $x_{1}(\mathrm{~s})$, $y_{1}(s) \in \mathcal{E}$ sont premiers si et seulement si $\exists x_{2}(s), y_{2}(s) \in \mathcal{E}$ tels que

$$
x_{1}(s) x_{2}(s)+y_{1}(s) y_{2}(s)=1 \text {. }
$$

Si $x_{1}(s), y_{1}(s) \in \mathcal{E}$ ne sont pas premiers, alors ils ont un plus grand facteur commun $z(s) \in \mathcal{E}$ et $\exists x_{2}(s), y_{2}(s) \in \mathcal{E}$ tels que

$$
x_{1}(s) x_{2}(s)+y_{1}(s) y_{2}(s)=z(s) .
$$

D'un point de vue pratique, il faut noter que le calcul des zéros ou facteurs commun à deux pseudopolynômes, i.e. des éléments de $\mathcal{E}$, est aisée, ainsi que la construction des pseudopolynômes qui interviennent dans l'identité de Bézout [4, 5]. Compte tenu des corollaires 3.7 et du théorème 3.9 , il est bien clair que l'introduction de l'anneau $\mathcal{E}$ conduit à une méthodologie pour la stabilisation des systèmes à retards commensurables. En fait, elle permet d'assigner librement, dans le cas où il n'y a pas de zéros communs entre le numérateur et le dénominateur du transfert considéré, le dénominateur du système en boucle fermée. La deuxième condition du corollaire 3.7 impose simplement que le degré $n$, par rapport à la variable $s$, de ce dénominateur ne peut pas être diminué. A cette condition près, le placement arbitraire des pôles du système bouclé est possible. C'est une méthode de stabilisation connue pour sa robustesse. Dans le cas des systèmes à retards, elle présente de plus l'avantage d'éviter la vérification de la stabilité du système bouclé. Il est en effet difficile de vérifier si oui ou non un polynôme en $s$, $\mathrm{e}^{\theta s}$ possède des zéros à partie rélle positive, sauf bien sûr dans le cas où le polynôme à tester, qui est le 
dénominateur choisi du système en boucle fermée, est simplement un polynôme en $s$.

Nous renvoyons le lecteur intéressé par de plus amples détails à [3, 5, 22]. Avant d'illustrer l'emploi de cette technique dans le cas de l'équation de la chaleur, il nous faut encore aborder les difficultés posées par les systèmes à retards de type neutre.

\subsection{LE CAS DES SYSTÈMES DE TYPE NEUTRE} $e^{\theta s}$

Le dénominateur d'un système à retards commensurables est un polynôme en $s$,

$$
\hat{A}(s)=\sum_{i=0}^{n} \sum_{k=0}^{q} A_{i k} s^{i} \mathrm{e}^{k \theta s} .
$$

Dans le cas d'un système causal, le terme principal [30] $A_{n q}$ n'est jamais nul. On parle de système à retard proprement dit lorsque $A_{n k}=0,0 \leq k \leq q-1$, et on parle de système de type neutre dans le cas contraire. Un système de type neutre est dit formellement stable lorsque tous les zéros du polynôme principal

$$
p\left(\mathrm{e}^{\theta s}\right)=\sum_{k=0}^{q} A_{n k} \mathrm{e}^{k \theta s}
$$

sont stables, à partie réelle strictement négative.

La méthode de stabilisation des systèmes à retards à l'aide de retards distribués, basée sur les propriétés de l'anneau $\mathcal{E}$, s'adapte au cas des systèmes de type neutre formellement stable [2]. Elle permet d'assigner au système en boucle fermée un dénominateur de la forme

$$
\hat{X}(s)=p^{m}\left(\mathrm{e}^{\theta s}\right) r(s),
$$

où $r(s)$ est un polynôme en $s$ de degré $n, p\left(\mathrm{e}^{\theta s}\right)$ est le polynôme principal du dénominateur du système en boucle ouverte et $m \in \mathbb{N}$.

Il est clair que cette méthode ne permet pas de stabiliser un système de type neutre non formellement stable. On peut montrer [27] qu'il est impossible de stabiliser un tel système avec une loi de commande qui est elle-même un système à retards, même en utilisant des retards distribués. La raison en est simple. L'equation (3.4) montre que le polynôme principal du système bouclé est le produit des polynômes principaux du système et de la loi de commande. Ainsi le système bouclé est nécessairement non formellement stable, et donc instable, lorsque le système en boucle ouverte est lui-même non formellement stable.

Dans ce cas l'emploi d'une loi de commande prenant en compte non seulement la mesure de la sortie, mais aussi sa dérivée permet, dans certains cas, de stabiliser le système $[6,27]$.

Nous emploierons aussi cette technique dans le cas de l'équation de la chaleur.

\section{Stabilisation de L’Équation de la chaleur}

Rappellons que le système que nous cherchons à stabiliser s'écrit

$$
\begin{aligned}
\hat{y}(s) & =\hat{H}(s) \hat{u}(s) \\
& =\frac{2 \mathrm{e}^{-h l \sqrt{s}}}{h \sqrt{s}\left(1-\mathrm{e}^{-2 h l \sqrt{s}}\right)} \hat{u}(s)
\end{aligned}
$$

On note, à partir d'un développement en série, que ce système possède un pôle à $s=0$, et qu'il est donc instable ${ }^{1}$. Nous l'avions déja constaté au $§ 3.2$. En liaison

1. La barre étant isolée, elle se comporte comme un accumulateur de chaleur. C'est donc un intégrateur pour les basses fréquences.

ESAIM: Proc., VOL. 5, 1998, 131-144 
avec les rappels précédents, on peut factoriser ce transfert comme une fraction d'éléments de $\hat{\mathcal{R}}(\sigma)$

$$
\hat{H}(s)=\hat{N}(s) \hat{D}^{-1}(s)
$$

avec

$$
\begin{aligned}
\hat{N}(s) & =\frac{2 \mathrm{e}^{-h l \sqrt{s}}}{\sqrt{s}+\alpha} \\
\hat{D}(s) & =\frac{h \sqrt{s}\left(1-\mathrm{e}^{-2 h l \sqrt{s}}\right)}{\sqrt{s}+\alpha}
\end{aligned}
$$

où $\alpha>0$, de façon à ne pas introduire de zéro commun instable entre le numérateur et le dénominateur.

\subsection{Méthode directe}

On peut d'abord essayer une loi de commande simple, dite proportionnelle, définie par

$$
u(t)=\kappa(v(t)-y(t)),
$$

où $\kappa \in \mathbb{R}$. Le dénominateur du transfert du système en boucle fermée est alors

$$
\hat{X}(s)=h \sqrt{s}\left(1-\mathrm{e}^{-2 h l \sqrt{s}}\right)+2 \kappa \mathrm{e}^{-h l \sqrt{s}}
$$

Il est pratique de poser $z=h l \sqrt{s}$, de sorte que

$$
l \hat{X}(s)=z\left(1-\mathrm{e}^{-2 z}\right)+2 l \kappa \mathrm{e}^{-z}
$$

Posant $z=x+j y$, la recherche des zéros de $\hat{X}(s)$ se ramène au système

$$
\begin{cases}x \sinh x \cos y-y \cosh x \sin y+l \kappa & =0 \\ y \sinh x \cos y+x \cosh x \sin y & =0\end{cases}
$$

d'où l'on déduit en particulier

$$
x \cos ^{2} y-l \kappa \sinh x \cos y-x \cosh ^{2} x=0
$$

puis

$$
\cos y=\frac{l \kappa \sinh x \pm \sqrt{l^{2} \kappa^{2} \sinh ^{2} x+4 x^{2} \cosh ^{2} x}}{2 x}
$$

La plus grande valeur du membre de droite est plus grande que $\frac{l \kappa}{2}+1>1$, qui ne peut pas égaler cos $y$. L'autre solution est négative, et peut donc correspondre à une solution pour laquelle $|y| \geq \frac{\pi}{2}$. Il suffit que $x<\frac{\pi}{2}$ pour assurer l'absence de zéro de $\hat{X}(s)$ dans la partie droite du plan complexe. On montre finalement que ce résultat est obtenu en choisissant $0<\kappa<\frac{\pi}{2 l} \sinh \frac{\pi}{2}$, soit environ $0<\kappa<7 / l$.

Finalement on peut constater que le système bouclé n'a pas non plus de singularité dans la partie droite du plan complexe, pas même à l’infini du fait que

$$
\lim _{|s| \rightarrow \infty, \operatorname{Res} \geq 0} \frac{\hat{X}(s)}{\sqrt{s}+\alpha}=h \neq 0 .
$$

Il est donc stable.

Il est clair que ces calculs ne forment pas une méthode générale. On peut difficilement imaginer appliquer cette technique sur un transfert plus complexe. Notons qu'une méthodologie pour la synthèse de loi de commande de ce type stabilisant des transferts en $s^{\alpha}, \mathrm{e}^{\theta s}$ a récemment été décrite [16]. 


\subsection{Stabilisation par Un Bouclage DÉfini PaR UNE CONVOLUTion}

Lorsqu'on écrit le transfert de l'équation de la chaleur sous la forme

$$
\hat{T}(z)=\frac{2 l \mathrm{e}^{-z}}{z\left(1-\mathrm{e}^{-2 z}\right)},
$$

il pourrait sembler que l'on va se trouver confronter à un système de type neutre non formellement stable, que l'on ne pourra pas stabiliser en utilisant l'anneau $\mathcal{E}$. En fait il n'en est rien. La différence, fondamentale, est que dans le cas présent

$$
\lim _{|s| \rightarrow \infty, \operatorname{Res} \geq 0}\left(1-\mathrm{e}^{-2 h l \sqrt{s}}\right)=1
$$

alors que

$$
1-\mathrm{e}^{-2 z}
$$

n'a pas de limite définie lorsque $|z| \rightarrow \infty$, Re $\geq 0$. Ces exemples illustrent bien l'intérêt du théorème 3.3 .

C'est pourquoi nous allons pouvoir utiliser la méthode suggérée au $§ 3.7$ pour stabiliser l'équation de la chaleur. Notons simplement que

$$
\begin{aligned}
& h \sqrt{s}\left(1-\mathrm{e}^{-2 h l \sqrt{s}}\right)\left(1+\alpha\left(\frac{1-\mathrm{e}^{-h l \sqrt{s}}}{\sqrt{s}}\right)^{2}\right) \\
& +2 h \alpha \mathrm{e}^{-h l \sqrt{s}}\left(\frac{1-\mathrm{e}^{-2 h l \sqrt{s}}}{\sqrt{s}}\right)\left(1-\frac{\mathrm{e}^{-h l \sqrt{s}}}{2}\right) \\
& =h\left(\frac{1-\mathrm{e}^{-2 h l \sqrt{s}}}{\sqrt{s}}\right)(s+\alpha),
\end{aligned}
$$

pour tout réel $\alpha$, de sorte que la loi de commande définie par

$$
\begin{aligned}
\hat{u}(s)= & -\alpha\left(\frac{1-\mathrm{e}^{-h l \sqrt{s}}}{\sqrt{s}}\right)^{2} \hat{u}(s) \\
& +h \alpha\left(\frac{2-\mathrm{e}^{-h l \sqrt{s}}-2 \mathrm{e}^{-2 h l \sqrt{s}}+\mathrm{e}^{-3 h l \sqrt{s}}}{2 \sqrt{s}}\right)(\hat{v}(s)-\hat{y}(s)),
\end{aligned}
$$

avec $\alpha>0$, stabilise le système. L'expression temporelle de cette relation est la suivante.

$$
\begin{aligned}
u(t)= & -\alpha \int_{0}^{t}\left(1-2 \operatorname{erfc}\left(\frac{h l}{2 \sqrt{\tau}}\right)+\operatorname{erfc}\left(\frac{h l}{\sqrt{\tau}}\right)\right) u(t-\tau) d \tau \\
& +h \alpha \int_{0}^{t} \frac{2-\mathrm{e}^{-\frac{h^{2} l^{2}}{4 \tau}}-2 \mathrm{e}^{-\frac{h^{2} l^{2}}{\tau}}+\mathrm{e}^{-\frac{9 h^{2} l^{2}}{4 \tau}}}{2 \tau \sqrt{\pi \tau}}(v(t-\tau)-y(t-\tau)) d \tau .
\end{aligned}
$$

Par cette loi de commande, on a fait glisser le pôle instable en $s=0$ du système en boucle ouverte vers un pôle stable en $s=-\alpha$ du système en boucle fermée. Les autres pôles du système, qui sont en

$$
s=-\frac{k^{2} \pi^{2}}{h^{2} l^{2}},
$$

pour $k \in \mathbb{N}^{*}$, sont stables et restent inchangés sous l'action de cette loi de commande. 


\subsection{Stabilisation par un bouclage sur la dérivée de la sortie}

Notons maintenant que

$$
\begin{aligned}
& h \sqrt{s}\left(1-\mathrm{e}^{-2 h l \sqrt{s}}\right)\left(1+\alpha\left(\frac{1-\mathrm{e}^{-h l \sqrt{s}}}{\sqrt{s}}\right)\right) \\
& +2 h \mathrm{e}^{-h l \sqrt{s}}\left(s \frac{\mathrm{e}^{-2 h l \sqrt{s}}}{2 \sqrt{s}}+\frac{\alpha}{2}\left(1+\mathrm{e}^{-h l \sqrt{s}}-\mathrm{e}^{-2 h l \sqrt{s}}\right)\right) \\
& =h(\sqrt{s}+\alpha),
\end{aligned}
$$

pour tout réel $\alpha$, de sorte que la loi de commande définie par

$$
\begin{aligned}
\hat{u}(s)= & -\alpha\left(\frac{1-\mathrm{e}^{-h l \sqrt{s}}}{\sqrt{s}}\right) \hat{u}(s)-h \frac{\mathrm{e}^{-h l \sqrt{s}}}{2 \sqrt{s}} s \hat{y}(s) \\
& +\frac{h \alpha}{2}\left(1+\mathrm{e}^{-h l \sqrt{s}}-\mathrm{e}^{-2 h l \sqrt{s}}\right)(\hat{v}(s)-\hat{y}(s)),
\end{aligned}
$$

avec $\alpha>0$, stabilise le système. L'expression temporelle de cette loi de commande est

$$
\begin{aligned}
u(t)= & \int_{0}^{t} \frac{\alpha}{\sqrt{\pi \tau}}\left(\mathrm{e}^{-\frac{h^{2} l^{2}}{4 \tau}}-1\right) u(t-\tau) d \tau \\
& +\int_{0}^{t} \frac{h}{2 \sqrt{\pi \tau}} \mathrm{e}^{-\frac{h^{2} l^{2}}{4 \tau}} \dot{y}(t-\tau) d \tau \\
& +\int_{0}^{t} \frac{h \alpha}{2}\left(\frac{h l}{2 \tau \sqrt{\pi \tau}} \mathrm{e}^{-\frac{h^{2} l^{2}}{4 \tau}}-\frac{h l}{\tau \sqrt{\pi \tau}} \mathrm{e}^{-\frac{h^{2} l^{2}}{\tau}}\right)(v(t-\tau)-y(t-\tau)) d \tau \\
& +\frac{h \alpha}{2}(v(t)-y(t)) .
\end{aligned}
$$

Nous n'abordons pas dans cette communication la question difficile du calcul numérique des lois de commande définies de cette manière. L'approche diffusive, présentée dans [25], répond à cette question, autorisant ainsi leur mise en oeuvre.

\section{RÉFÉRENCES}

[1] R. Bellman, K.L. Cooke: Differential-Difference Equations, Academic Press, New York, 1963.

[2] D. Brethé: Stabilization and observation of neutral-type delay systems, In Proc. IFAC Conf. Industrial Systems, 353-358, Belfort, 1997.

[3] D. Brethé: Contribution à l'étude de la stabilisation des systèmes linéaires à retards, Thèse de Doctorat, École Centrale de Nantes-Université de Nantes, 1997.

[4] D. Brethé, J.J. Loiseau: A result that could bear fruit for the control of delay-differential systems, In Proc. 4th IEEE Mediteranean Symp. Control and automation, Chania, Greece, 168-172, 1996.

[5] D. Brethé, J.J. Loiseau: An effective algorithm for finite spectrum assignment of single-input systems with delays, Math. Computer in Simulations, Special issue on time-delay systems, V. Kolmanovskii and J.P. Richard Eds., 1997.

[6] C.I. Byrnes, M.W. Spong, T.-J. Tarn: A several complex variables approach to feedback stabilization of linear neutral delay-differential systems, Math. Systems Theory, 17, 97-133, 1984.

[7] F.M. Callier, C.A. Desoer: An algebra of transfer functions for distributed linear timeinvariant systems, IEEE Trans. Circuits and Systems, 25, 9, 651-662, 1978.

[8] C.A. Desoer, M. Vidyasagar: Feedback systems: Input-output properties, Academic Press, New York, 1975.

[9] G. Doetsch: Introduction à l'utilisation pratique de la transformation de Laplace, GauthierVillars, Paris, 1959.

[10] M. Fliess et H. Mounier: Controllability and observability of linear delay systems: an algebraic approach, COCV (Control, Optimisation and Calculus of Variations), 3, 301-314, 1998.

[11] M. Fliess, H. Mounier, P. Rouchon et J. Rudolph: Systèmes linéaires sur les opérateurs de Mikusiński et commande d'une poutre flexible, ESAIM: Proceedings, 2, 183-193, 1997. 
[12] M. Fliess, H. Mounier, P. Rouchon et J. Rudolph: Tracking control of a vibrating string with an interior mass viewed as delay system, COCV (Control, Optimisation and Calculus of Variations), 3, 315-321,1998.

[13] H. Glüsing-Lüerßen: A behavioral approach to delay-differential systems, SIAM J. Control Opt., 35, 480-499, 1997.

[14] E. Hille, R.S. Phillips: Functional analysis and semigroups, Amer. Math. Soc. Coll. Publ., XXXI, New Providence, 1957.

[15] J. Hladik: La transformation de laplace à plusieurs variables, Masson \& Cie, Paris, 1969.

[16] R. Hotzel: Contribution a la théorie structurelle et à la commande des systèmes linéaires fractionnaires, Thèse de Doctorat, Supélec, Gif-sur-Yvette, 1998.

[17] E. Kamen: On an algebraic theory of systems defined by convolution operators, Math. System Theory, 9, 57-74, 1975.

[18] E. Kamen: An operator theory of linear functional differential equations, J. Differential Equations, 27, 274-297, 1978.

[19] E.W. Kamen, P.P. Khargonekar, A. Tannenbaum: Proper stable bezout factorizations and feedback control of linear time-delay systems, Int. J. Cont., 43, 837-857, 1986.

[20] V.B. Kolmanovskii, V.B. Nosov: Stability of functional differential equation, Academic Press, New York, 1986.

[21] V. Kučera: Discrete linear control, the polynomial equation approach, Wiley, Chichester, 1979

[22] J.J. Loiseau: Algebraic tools for the control and stabilization of time-delay systems, In Proc. IFAC Workshop LTDS, Grenoble, France, 1998.

[23] A.Z. Manitius, A.W. Olbrot: Finite spectrum assignment problem for systems with delays, IEEE Trans. Automatic Control, 35, 541-553, 1979.

[24] D. Matignon. Stability results for fractional differential equations with applications to control processing. In Computational Engineering in Systems Applications, volume 2, pages 963-968, Lille, France, July 1996. IMACS, IEEE-SMC.

[25] G. Montseny: Diffusive representation of pseudo-differential time-operators, in this volume.

[26] H. Mounier: Propriétés structurelles des systèmes linéaires à retards: aspects théoriques et pratiques, Thèse de Doctorat, Supélec, Gif-sur-Yvette, 1995.

[27] D.A. O'Connor, T.J. Tarn: On stabilization by state feedback for neut ral differential difference equations, IEEE Trans. Automatic Control, 28, 615-618, 1983.

[28] A.W. Olbrot: On controllability of linear systems with time delays in the control, IEEE Trans. Automatic Control, 16, 664-666, 1972.

[29] L. Pandolfi: Dynamic stabilization of systems with input delays, Automatica, 27, 1047-1050, 1991

[30] L.S. Pontryagin: On the zeros of some elementary transcendental functions, Izvestiya Akademii Nank SSSR, 6, 115-131, 1942.

[31] H.H. Rosenbrock: State-space and multivariable theory, Nelson, London, 1970.

[32] L. Schwartz: Théorie des distributions, Hermann, Paris, 1966.

[33] Y. Takahashi, M.J. Rabins, D.M. Auslander: Control and dynamic systems, Addison-Wiley, Reading, Ma., 1970.

[34] M. Vidyasagar: Control system synthesis, a factorization approach, MIT Press, Cambridge, MA, 1985.

[35] W.M. Wonham: Linear multivariable control: A geometric approach, Applications of Mathematics, Vol. 10, Springer Verlag, New York, 1979 